Planetary Systems in the Universe - Observation, Formation and Evolution

Proceedings IAU Symposium No. 202, (C)2004 IAU

Alan Penny, Pawel Artymowicz, Anne-Marie Lagrange, 8 Sara Russell, eds.

\title{
Modal structure of earth-like planets in the presence of magnetic field
}

\author{
S. Nasiri \\ Institute for Advanced Studies in Basic Scinces P. O. Box 45195-159, \\ Zanjan, Iran. Department of Physics, Zanjan University, Zanjan, Iran
}

\begin{abstract}
Free oscillations of the earth received much attention by the pioneering work of Benioff (1954). He observed an oscillation period of 57 minutes by investigating the data of Kamchatka earthquake and assumed the free oscillations of the earth as its origin. Since then many aspects of the problem has been investigated by different authors.
\end{abstract}

The mathematical technique which is used here to study and classify the possible motions of the earth is first proposed by Cowling(1941) and extended by others in studying the fluids oscillations. In this method, the free oscillations of heavenly fluid bodies are classified into acoustic and gravity $\operatorname{modes}(p$ and $g$ modes in their jargon, respectively). The $p$ modes are generated mainly by pressure fluctuations while the $g$ modes are mainly due to density fluctuations (See Sobouti, 1977). In a medium consisting of liquid and solid, in addition to the pressure and buoyancy forces arising in liquid part, the shear forces are also allowed by solid part. Therefore, a third category of modes, i.e., the toroidal modes are emerged. These are pure toroidal motions without coupling with everpresent $p$ and $g$ modes. Such a model for earth with liquid core and solid mantle is considered by Sobouti(1995) using the same technique and the possible motions are classified assuming a polytropic stratification for the model. Abedini(1999) used the same method to study the free oscillations of the earth by employing its real parameters given by Gilbert et al (1975). Furthermore, the oscillations of a magnetized fluid is studied by Sobouti(1977b), Nasiri et al(1989) and Nasiri(1992). In the presence of magnetic field the toroidal modes are excited resulting the hydromagnetic waves. Also, the everpresent $p$ and $g$ modes are coupled with $t$ modes. The coupling is proportional to the strength of the magnetic field.

We consider an isotropic system with a solid inner core and liquid outer core and solid mantel in equilibrium. Assume $\rho(r), P(r), U(r)$, and $\mu(r)$ be the density, the pressure, the gravitational potential, and the rigidity coefficient, respectively. The medium is pervaded by a force free magnetic field $\mathbf{B}$. Let the system undergo a small perturbation, and a mass element at position $\mathbf{r}$ be displaced by a small distance, $\xi(\mathbf{r}, t)$. Associated with this displacement are the Eulerian changes $\delta \rho, \delta P, \delta U$, and $\delta B$. It can be shown that the linearized equation of motion is

$$
\rho \frac{\partial^{2} \xi}{\partial t^{2}}=\mathcal{W} \xi
$$


where

$$
\begin{aligned}
\mathcal{W}_{i j} \xi & =\frac{\partial}{\partial x_{i}} \delta P+\delta \rho \frac{\partial U}{\partial x_{i}}+\rho \frac{\partial}{\partial x_{i}} \delta U \\
& -\frac{1}{4 \pi} \epsilon_{i j k} \epsilon_{j l m}\left[\frac{\partial}{\partial x_{l}}(\delta B)_{m} B_{k}+\frac{\partial}{\partial x_{l}} B_{m}(\delta B)_{k}\right] \\
& -\frac{\partial}{\partial x_{j}}\left[\mu\left(\frac{\partial \xi_{i}}{\partial x_{j}}+\frac{\partial \xi_{j}}{\partial x_{i}}-\frac{2}{3} \frac{\partial \xi_{r}}{\partial x_{r}} \delta_{i j}\right]\right.
\end{aligned}
$$

and

$$
\begin{gathered}
\delta \rho=-\rho \frac{\partial \xi_{i}}{\partial x_{i}}-\xi_{i} \frac{\partial \rho}{\partial x_{i}} \\
\delta P=-\gamma P \frac{\partial \xi_{i}}{\partial x_{i}}-\xi_{i} \frac{\partial P}{\partial x_{i}} \\
\delta B_{i}=\epsilon_{i j k} \epsilon_{k l m} \frac{\partial\left(\xi_{l} B_{m}\right)}{\partial x_{i}} \\
\frac{\partial^{2}(\delta U)}{\partial x_{i} \partial x_{i}}=4 \pi G \delta \rho
\end{gathered}
$$

where the summation over repeated indices is implied. $\gamma$ is the ratio of specific heats that can be obtained by assuming an appropriate equation of state for the earth's interior structure. The equation of state used here is

$$
P=\frac{3}{2} k_{0}\left[\left(\frac{\rho}{\rho_{0}}\right)^{\frac{7}{3}}-\left(\frac{\rho}{\rho_{0}}\right)^{\frac{5}{3}}\right]
$$

where $k_{0}$ and $\rho_{0}$ are bulk modulus and density on the earth surface, respectively. Using Eq. (7) and assuming adiabatic processes one can obtain the following expression for $\gamma$ (Abedini, 1999)

$$
\gamma=\frac{\frac{7}{3}\left(\frac{\rho}{\rho_{0}}\right)^{\frac{7}{3}}-\frac{5}{3}\left(\frac{\rho}{\rho_{0}}\right)^{\frac{5}{3}}}{\left(\frac{\rho}{\rho_{0}}\right)^{\frac{7}{3}}-\left(\frac{\rho}{\rho_{0}}\right)^{\frac{5}{3}}}
$$

We assume an axisymmetric force free magnetic field consisting of toroidal and poloidal components as follows(Ferraro and Plumton, 1966)

$$
\mathbf{B}=B_{0}\left[\frac{n(n+1)}{\eta r} Z_{n}(\eta r) Y_{n}(\theta), \frac{1}{\eta}\left(\frac{d}{d r}+\frac{1}{r}\right) Z_{n}(\eta r) \frac{d Y_{n}(\theta)}{d \theta}, Z_{n}(\eta r) \frac{d Y_{n}(\theta)}{d \theta}\right],
$$

where $Z_{n}(\eta r)=\left(\frac{\pi}{2 \eta r}\right)^{1} 2 J_{n+1 / 2}(x)$, is a spherical Bessel function and $Y_{n}(\theta)$ is a spherical harmonic. Assuming appropriate boundary conditions on the magnetic field gives $\eta r$ as a zero of the Bessel function (Nasiri, 1989). Hereafter we use the first order Bessel function, $n=1$, and its first zero, $\eta r=4.493409$. The force free nature of the field would keep the equilibrium configuration of the system spherically symmetric. Assuming an exponential time dependence, $e^{i \omega t}$, for $\xi$ we multiply Eq. (1) by $\xi^{*}$ and integrate over the medium. Using a modified form of Helmholtz theorem (Sobouti, 1981) we decompose a lagrangian displacement 
into $p, g$ and $t$ motions and using the Rayleigh-Ritz variational technique to minimize the eigenvalues one gets a matrix form for Eq. (1). The matrix equation is solved numerically to obtain the eigenvalues and the variational parameters as eigenfunctions. The eigenvalues of $p$ modes does not change considerably in the presence of the magnetic field. The eigenvalues of $g$ modes, $\omega_{g}^{2}$, for whole earth and also for outer liquid core of the earth are as follows.

\begin{tabular}{lcccccc}
\hline \multirow{2}{*}{ radial mode number } & & 1 & 2 & 3 & 4 & 5 \\
\hline \multirow{3}{*}{ for earth } & $\lambda=0.0$ & 39.961 & 15.370 & 2.314 & 0.617 & 0.124 \\
& $\lambda=0.1$ & 39.992 & 15.876 & 2.455 & 0.802 & 2.314 \\
\hline \multirow{3}{*}{ liquid core } & $\lambda=0.0$ & -0.398 & -0.124 & -0.061 & -0.028 & -0.006 \\
& $\lambda=0.1$ & -0.392 & -0.109 & -0.021 & 0.010 & 2.424 \\
\hline
\end{tabular}

Table 1. The $g$ eigenvalues $\left(\omega_{g}^{2}\right)$, in unit of $\omega_{j}^{2}=\frac{P_{c}}{\rho R^{2}}$ for whole earth and liquid outer core for first five radial mode numbers, in the absence $(\lambda=0.0)$ and presence $(\lambda=0.1)$ of the magnetic field.

It is seen that, the decreasing characteristic of $\omega_{g}^{2}$ is not preserved in the presence of the magnetic field and they increase for higher mode orders for the whole earth. In outer liquid core, where the unstable convective motions take place due to negative values of $\omega_{g}^{2}$, the effect of magnetic field becomes more important. $\omega_{g}^{2}$ for higher mode orders changes sign to be positive indicating the stable modes in the presence of the magnetic field. Therefore, the convective motions would be essentially decelerated by the magnetic field. Could this effect be justified as a kind of "magnetic cooling" for the earth? Numerical values for $\omega_{t}^{2}$ are also computed. These modes are excited either by shear forces or by magnetic forces. So, they would be degenerate for nonmagnetized fluid core. The presence of magnetic field excites these modes producing the hydrom compared to the periods of the seismic waves propagated by shear forces in solid part of the earth.

\section{References}

Abedini, Y. 2000, Iranian J. Phys. Research, (in press)

Cowling, T. G. 1941, MNRAS, 101, 367

Ferraro, V. C. A., \& and Plumton, C., 1966, An Introduction to Magneto-Fluid Mechanics, 2nd ed. Clarendon Press, Oxford

Gilbert, F., \& Dziewonski, A. M. 1975, Philos. Trans. R. Soc., London Ser. A, 275, 187

Nasiri, S., \& Sobouti, Y. 1989, A\&A, 217, 127

Nasiri, S. 1992, A\&A, 261, 615

Sobouti, Y. 1977, A\&A, 55, 339

Sobouti, Y. 1981, A\&A, 100, 319

Sobouti, Y. 1995, Iranian J. Phys. Research, 1, 49 